\title{
USE OF ORTHOGONAL ARRAYS IN MECHANISM SYNTHESIS
}

\author{
SRIDHAR KOTA and SHEAN-JUINN CHIOU \\ Design Laboratory, Department of Mechanical Engineering and Applied Mechanies, \\ The University of Michigan. Ann Arbor, Michigan, U.S.A.
}

(Received IS November 1991; received for publication 5 November 1992)

\begin{abstract}
Abatract-No single optimization method exists that is best suited to solve all path generation problems effectively [Erdman 1991]. Graphical illustrations of the mechanism design space, given in this paper, altest to the dificulties encountered in the optimization of path generation tasks. An example has also been provided to illustrate the importance of a good initial guess in achieving proper convergence. Use of experimental design techniques that are based on statistically designed orthogonal arrays is suggested in this paper, as an alternate method to solve mechanism design tasks. It will also be beneficial to use this method to obtain a good starting point for the traditional optimal synthesis procedures. Also, when a theoretical solution is translated inlo physical reality, manufacturing tolerances, joint clearances, and elasticity in link members cause variations in mechanism performance. Orthogonal arrays and the related robust design procedures, suggested here, account for such variations as well. Design examples are given to illustrate the use of orthogonal arrays.
\end{abstract}

\section{INTRODUCTION}

\section{Optimization techniques}

The task of determining the optimal dimensions of a linkage that can pass through a set of desired points in a prescribed sequence has been addressed by several researchers in the past. The solution methods can be broadly classified into two categories: (1) precision point synthesis and (2) optimization. Chapters 2 and 11 of [Erdman 1991] provide an excellent history of developments in planar synthesis and optimization in mechanisms, respectively. Although closed form analytical precision point synthesis techniques guarantee that all the synthesized linkages pass precisely through a given set of points, only a limited number of points can be prescribed; up to five in the case of a four-bar linkage, with prescribed input timing. Jensen [Jensen 1984] presented a graphical method for synthesis of four-bar linkages for up to 12 points. Roth [Roth 1962] extended the Burmester theory to nine-point path generation using geared five-bar mechanisms. On the other hand, optimization techniques allow us to specify more points (10-20 or more) to describe a desired curve, but solution convergence is not always guaranteed. Proper choice of the starting point is critical to convergence of the solution for any optimization method and is especially so in the mechanism synthesis domain due to pronounced nonlinearities, nonmonotonic objective functions, and multiple local minima. While many optimization techniques have been successfully applied to different path generation tasks in the last four decades, their success has often been problemdependent. In 1973, Fox and Gupta surveyed various mechanism optimization methods and observed that no single method existed that is best suited to all problems. Almost two decades later, in 1991, Liu and Angeles' survey [Erdman 1991, Chap. 11] led to a similar observation. One notable contribution has been the Selective Precision Synthesis method developed by Kramer and Sandor in the early 1970's and since enhanced and applied to spatial mechanisms. In this method a realistic error envelope is defined around the mechanism precision points and solutions are found using various optimization methods.

\section{Tolerances}

Let us assume that a linkage has been synthesized analytically or otherwise to pass exactly through a set of desired points. When the design is translated into a physical reality, manufacturing tolerances, joint clearances, and elasticity in link members cause variations in the performance. The question then is "How exactly can a synthesized mechanism pass through the given points?" 
It is, therefore, important to account for the inevitable physical limitations and constraints while searching for "exact" analytical solutions. The effect of tolerances, joint clearances, and link deflections can be lumped together as an effective change in theoretical link length as the mechanism progresses from the design realm to manufacturing realm. Various analytical and statistical techniques have been developed to analyze the effect of tolerances and clearances [Knapp 1963; Grank and Fawcett 1977; Garret and Hall 1969; Kinzel and Hall 1975; Sharfi and Smith 1983] including computation of mechanism sensitivity coefficients [Faik and Erdman 1991]. A survey of tolerance and clearance analysis techniques is given in [Erdman 1991, Chap. 11]. In contrast to tolerance analysis, [Faik 1991] notes that, a more fundamental approach is to predict the sensitivity of mechanism solutions during the synthesis stage. [Faik and Erdman 1991] developed sensitivity maps to provide, a priori, the information on sensitivity of various solutions to link-length variations. These maps would allow the designer to weed out, early in the design process, solutions with inadequate sensitivity. A more efficient way is to consider link-length variations during the search process instead of weeding out inadequate solutions. This leads to robust designs, that is, designs that not only fulfill the performance requirements but are insensitive to variations due to tolerances and clearances. The experimental design techniques, presented in this paper, allow us to optimize both the nominal values of all design variables and the tolerances about these nominal values to generate robust solutions.

This paper addresses the following two issues that have been accepted in mechanisms design domain:

(1) there is no single optimization technique that can successfully be applied to all path generation tasks, and

(2) it is important to take into account variations in link-lengths during the synthesis process itself.

First, we will describe the nonlinear, nonmonotonic nature of the mechanism design space in support of the first statement by graphically illustrating the mechanism design space. We will introduce experimental design techniques using orthogonal arrays as a variable alternative to solving mechanism design problems. Experimental design does not necessarily mean that physical experiments are performed. Instead, it means that statistically designed orthogonal arrays are used to simultaneously investigate the effect of multiple design variables on the overall performance. In this paper, we will also illustrate, with a design example, how statistically designed experiments can be used not only to identify optimum link lengths but also to simultaneously account for variations in link-lengths due to tolerances, clearances, and link deflections. It is then followed by a brief description of a traditional optimal synthesis procedure based upon the Generalized Reduced Gradient whose intent is to illustrate the importance of having a good initial guess that would lead to proper convergence. The results of orthogonal array method are used as initial guesses for the GRG based method to show the improved convergence to an acceptable minimum. Thus, the usefulness of orthogonal array method in obtaining initial guesses for traditional and more efficient methods, is validated.

\section{BACKGROUND AND PROBLEM STATEMENT}

We focus our attention on the design of planar four-bar linkages for a given path generation task. We will assume that a desired curve is represented by a set of discrete points in $X-Y$ plane. A general four-bar linkage can be completely described by nine design variables (Fig. 1): two coordinates of the ground pivot $A_{0}$, lengths of the four links $L_{1}, L_{2}, L_{3}, L_{4}$, the angle of the input link $\theta_{2}$, and a vector (length $B P$ and angle $\gamma$ ) defining the path tracer point $P$. A wide variety of planar coupler curves can be generated by varying the design variables. It is widely known that the generated curves will be more orderly and symmetrical if $L_{3}=L_{4}=B P$, i.e. the symmetry condition. The dimensions of the design space are reduced, for the sake of simplicity, by taking advantage of this symmetry condition. We are interested in determining a linkage whose coupler curve matches the shape of the desired curve with minimum deviation. This means our objective function should be insensitive to variations in the size and orientation of the coupler curves. With such an objective function, we are no longer concerned with the actual location of the ground 


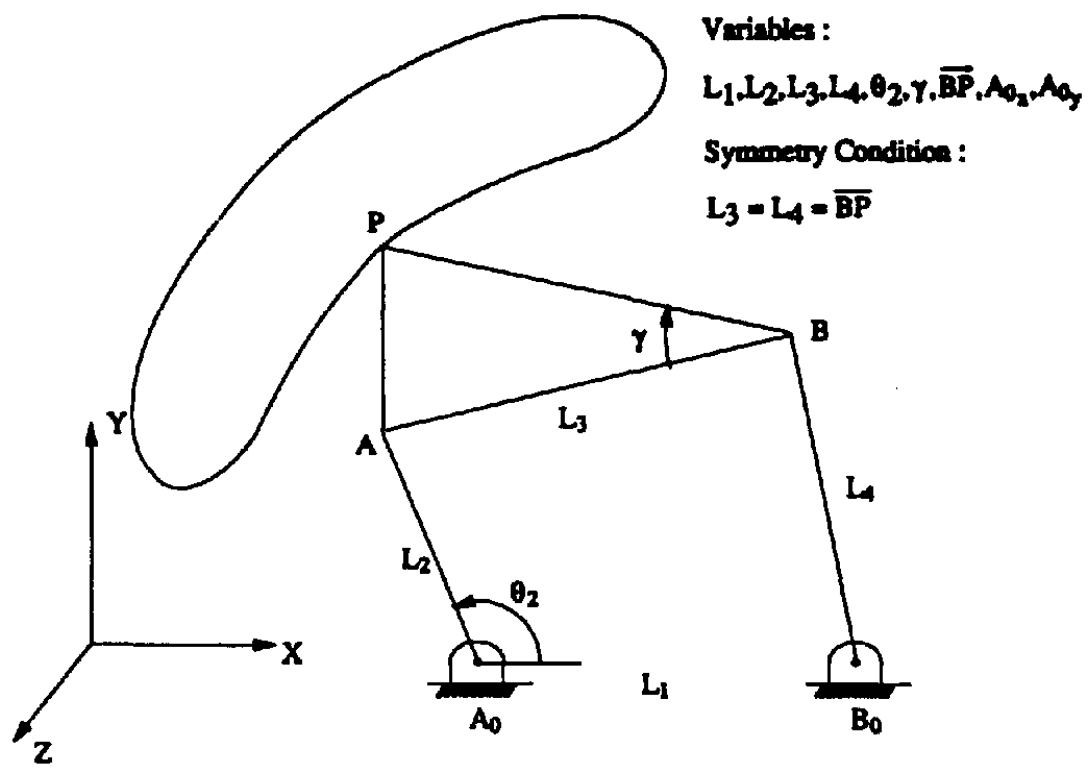

Fig. 1. A four-bar linkage with symmetry condition.

pivots. Consequently, we will require only four design variables to describe any four-bar linkage that traces symmetrical coupler curves. These four variables are: $L_{1}, L_{2}, L_{3}$ and $\gamma$. We have further reduced, without the loss of generality, the number of variables to three by taking the ratios $L_{1} / L_{2}$, $L_{3} / L_{2}$ and $\gamma$. Alternately, we can set the length of the input link $L_{2}=1$ unit. So the method used to compute the deviation should be independent of the size of the mechanism. This leads to a three-dimensional design space (Fig. 2) with the following three variables:

(1) Normalized common length: $L_{3} / L_{2}=L_{4} / L_{2}=B P / L_{2}$;

(2) Normalized length of the ground link: $L_{1} / L_{2}$;

(3) Coupler angle $\gamma$.

Necessitated by the simplifying assumptions made in reducing the dimension of the design space, we have developed a metric for comparing any two arbitrary curves irrespective of their orientation and size. This method is based on parametrization of given curves. The deviation between parametrized curves indicates the extent of similarity or dissimilarity between the two given curves irrespective of their size and orientation. The details of this parametrization method, reported in an earlier work [Kota 92], is briefly described ahead for the sake of completeness and for the immediate reference of the reader.

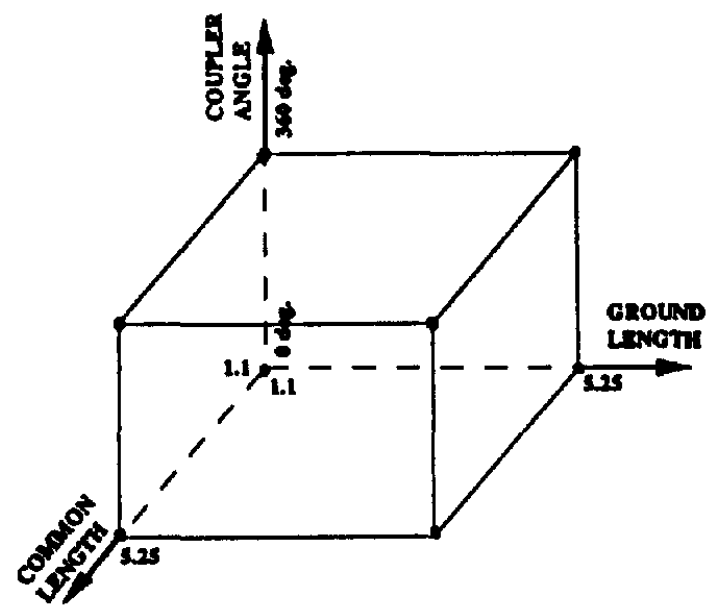

Fig. 2. Three dimensional design space for generating symmetrical coupler curves. 


\section{Computation of the deviation value}

In order to compute the deviation value or the similarity index, a set of points on the desired and the generated curve are necessary. The data points of the desired curve are provided by the designer. For a candidate curve selected from the design chart. a set of points are generated for $4^{2}$ increments of the input crank rotation. Using these points, the parameterization of both curves is then performed as follows.

The first step is to arbitrarily choose what is known as the starting point $P_{0}$ among the data points. However, if the curve is open, the starting point should be either of the end points. With this as the reference point two parameters which we call parametric length. PLENGTH and parametric angle, PANGLE are computed for every other point in the set. The computation of these parameters is illustrated in Fig. 3(a). (PLENGTH) is the ratio of the length of the line segment from $P_{0}$ and $P_{i}$ and the summation of all such lengths for other points. (PANGLE) is the angle between the tangents to the curve at point $P_{0}$ and $P_{1}$. The tangent at a point is calculated by generating a piecewise cubic curve through all the points with $C_{1}$ continuity.

By plotting (PANGLE) ${ }_{i}$ vs (PLENGTH), the parametric curve, such as the one shown Fig. 3(b), is generated for each curve. The deviation, $D$, is then computed as shown below.

$$
D=\sqrt{\sum_{i=1}^{n}\left(Y_{R i}-Y_{d t}\right)^{2}}
$$

where $Y_{s i}=Y$-coordinate of the parametric curve for the generated curve; $Y_{d t}=Y$-coordinate of the parametric curve for the desired curve; $n=$ number of points in the set.

For a realistic match between the two curves irrespective of their position and orientation in the 2D space, the starting point for both the curves should be analogous. This is accomplished by arbitrarily selecting the starting point on one curve, say on the desired curve, and $D$ is calculated for every point on the second curve as the starting point. The least value of $D$ among all of them is the correct value of the deviation. The impact of direction of traversal in generating the parametric curve for the second set of points, is also recognized and implemented. The deviation value computed using this method, although not very rigorous, is found to give a good measure of similarity between any two coupler curves. Devising a sophisticated algorithm will be given due consideration in future.

The task is to determine the dimensions of a four-bar linkage whose coupler curve "best" approximates a given desired curve. Our matching algorithm, described above, compares the desired curve against a coupler curve corresponding to a point in the design space of Fig. 2 and returns the deviation value that indicates how closely the two curves match in their shape. Therefore, our task is to determine the point in the three-dimensional design space that gives the minimum deviation value. Based on experience with many different examples, any deviation value of less than 0.075 units seemed like an acceptable match for most practical purposes especially when one considers the manufacturing tolerances. Of course, the smaller the better.

\section{DESIGN SPACE CHARACTERIZATION}

Identifying the point with minimum deviation value seems straightforward, and many optimization-search algorithms exist today to accomplish this task. However, examination of the nature of the design space reveals that the task is not as straightforward as it might at first seem. In order to investigate the behavior of the objective function, we have selected a linkage from the 3-D design chart and used its coupler curve as the desired curve. Our desired curve (or global minimum) corresponds to the coupler curve generated by the linkage with the following dimensions:

Common length 3.08 units;

Ground length 3.5 units;

Coupler angle $165.83^{\circ}$.

Figure 4 is a cross-sectional view of the three-dimensional design space where the cutting plane corresponds to a common length of 3.0 units. The coupler angle and ground length occupy the horizontal and vertical axes of the plane. The objective function is evaluated at various points in the three-dimensional design space, and the results are shown graphically in Fig. 5(a). Figure 5(b) 
(a)

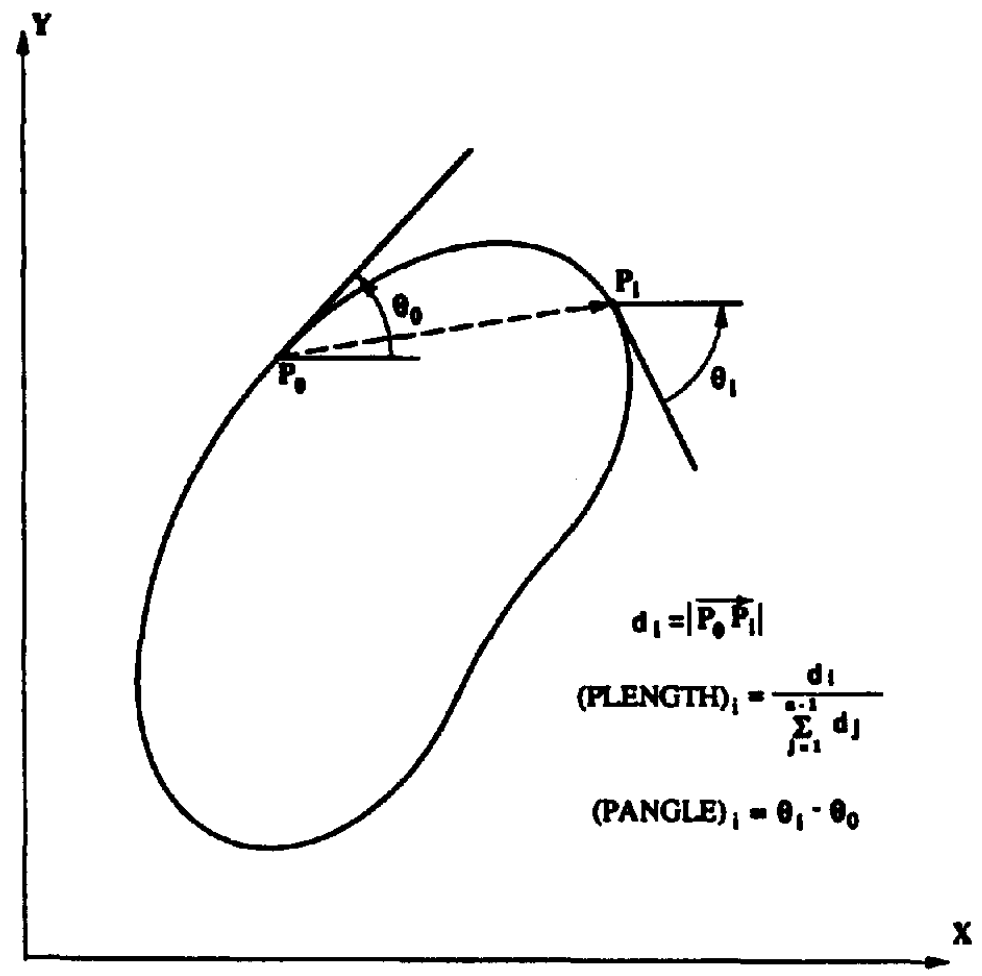

(b)

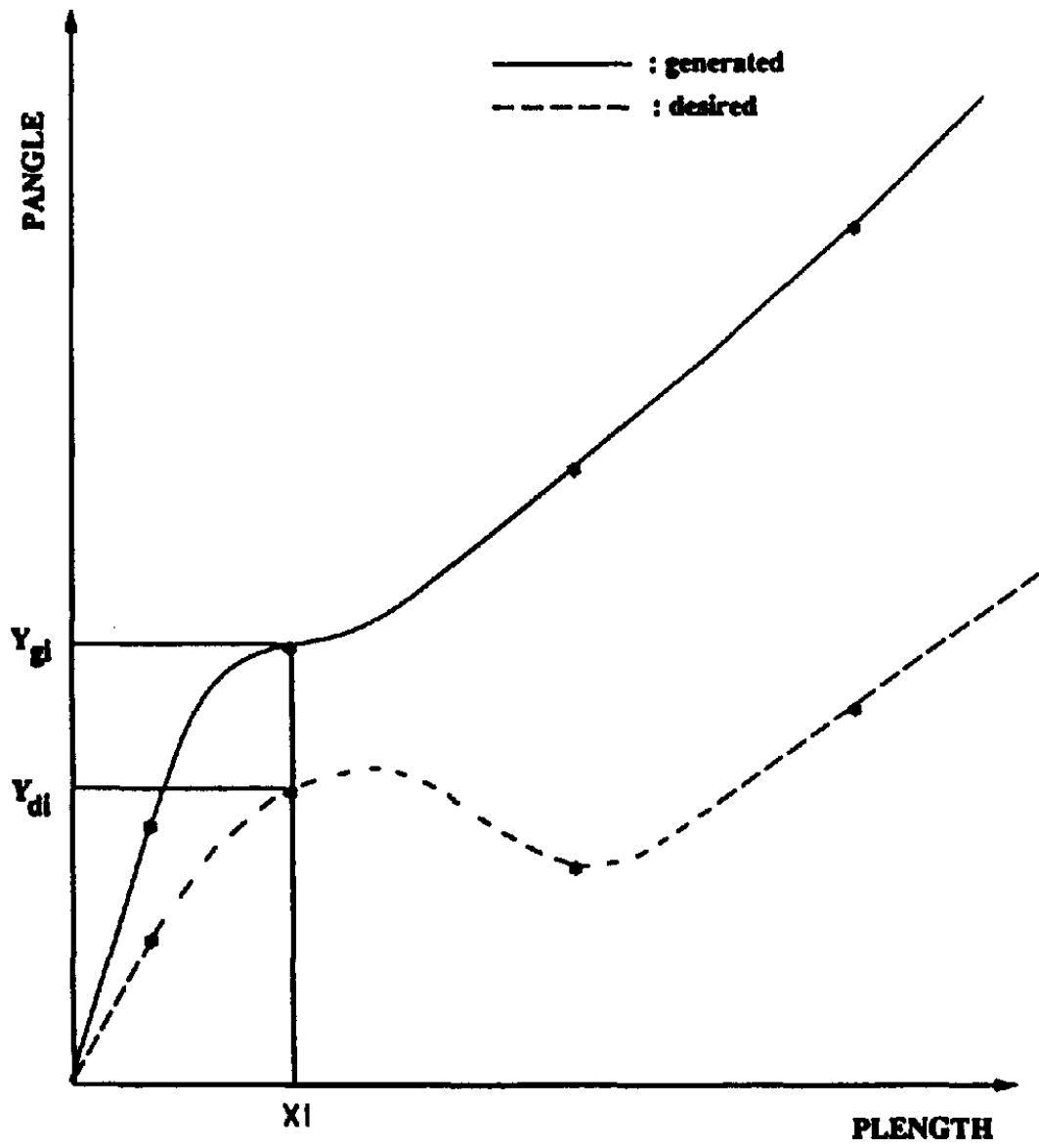

Fig. 3. (a) Computation of parametric kength (PLENGTH) and parametric angle (PANGLE). (b) Parametric curves for the desired and a generated curves. 


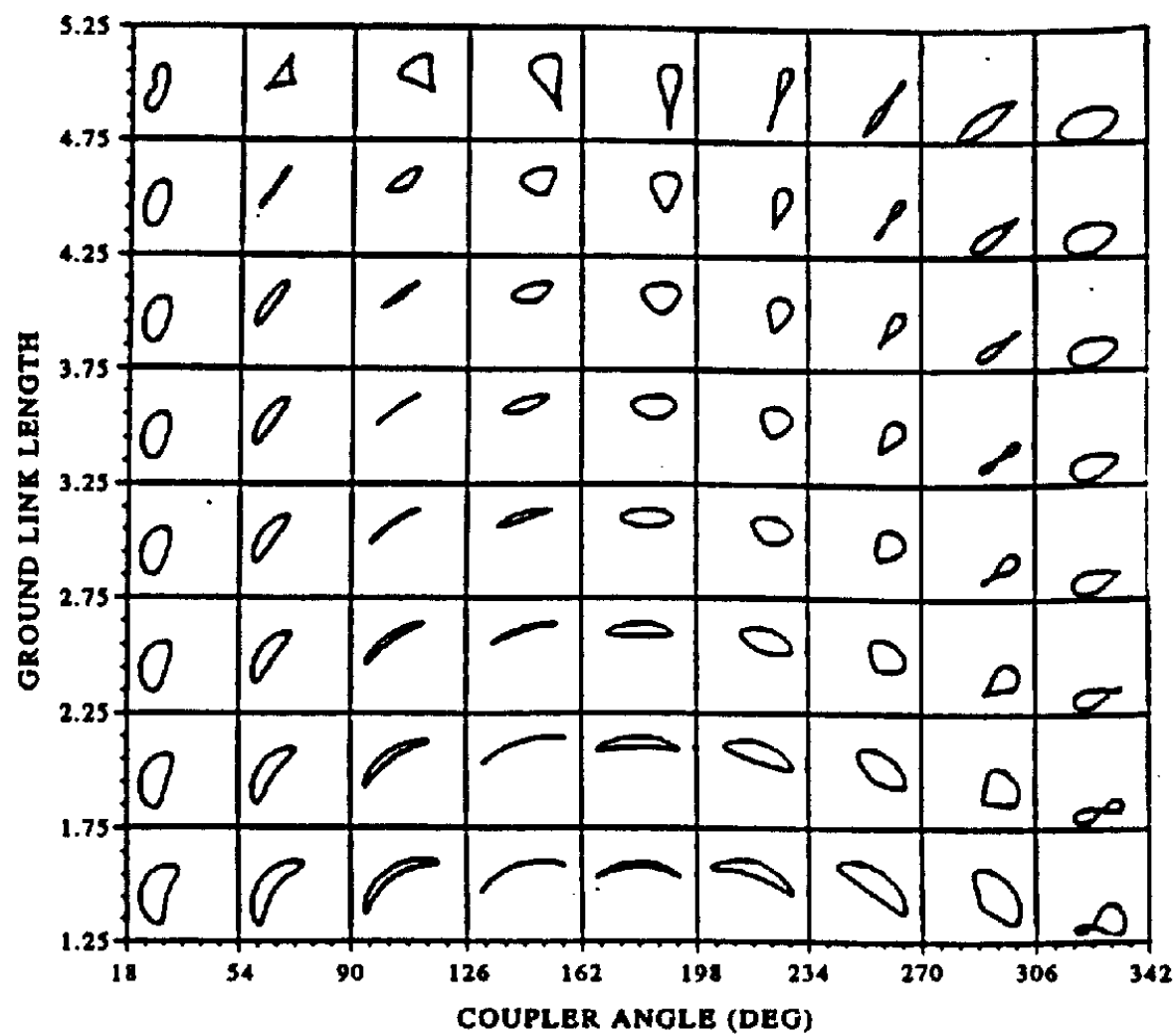

Fig. 4. A cross-sectional view of the three-dimensional design space shown in Fig. 3. The design "plane" shown here corresponds to common-length $=\mathbf{3 . 0}$ units.

shows the contour plot of the objective function for the design plane shown in Fig. 4. Figure S(c) shows the behavior of the objective function as a function of coupler angle and ground length when common length is fixed at 3.5 units. Figure 6 shows the behavior of the objective function as a function of coupler angle and common length with ground length fixed at 2.5, 3.0, 3.5, 4.0,

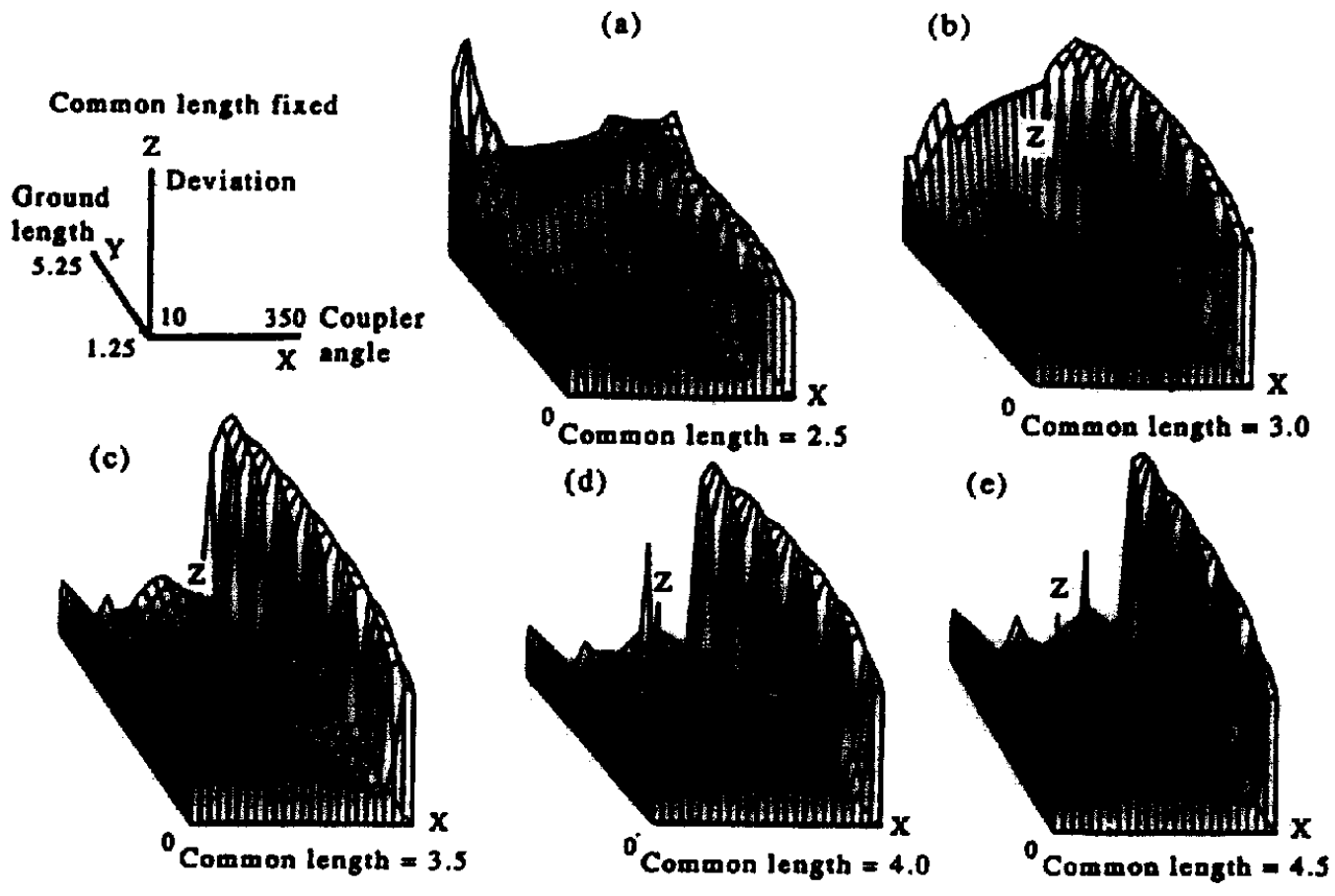

Fig. S. (Caption oppasite.) 
$\mathbf{z}$
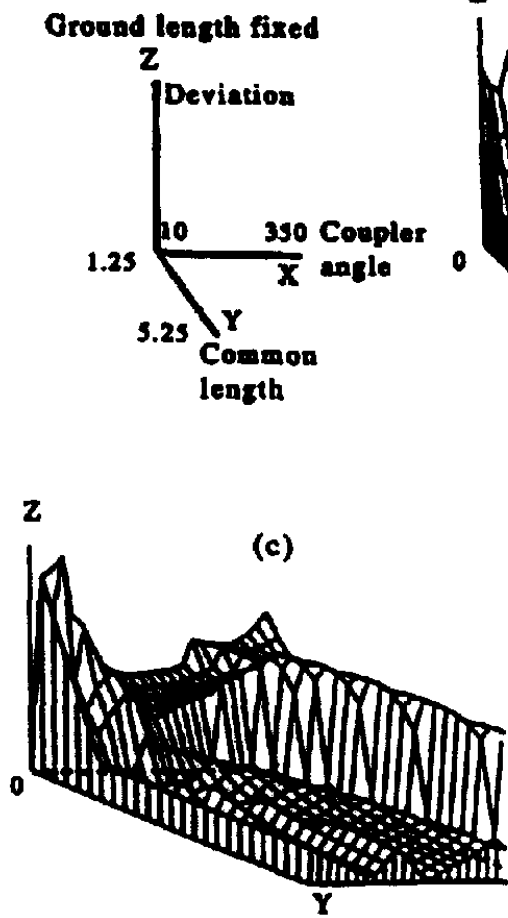

Ground length $=3.5$ (a)

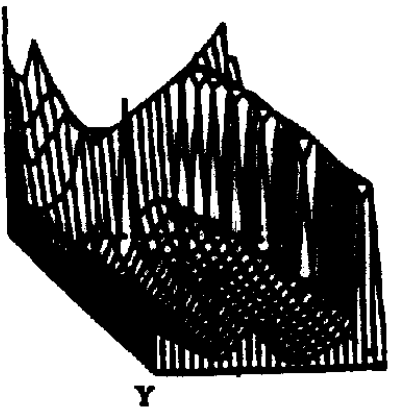

Ground length $=2.5$ z (b)

-

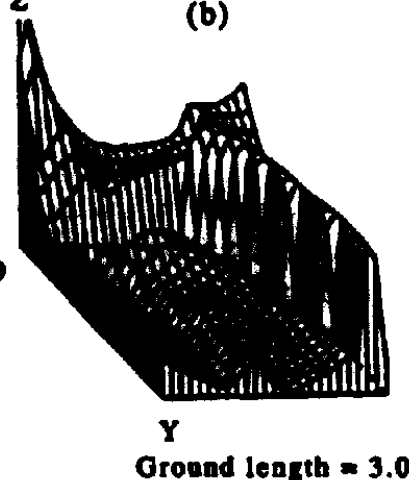

$\boldsymbol{z}$
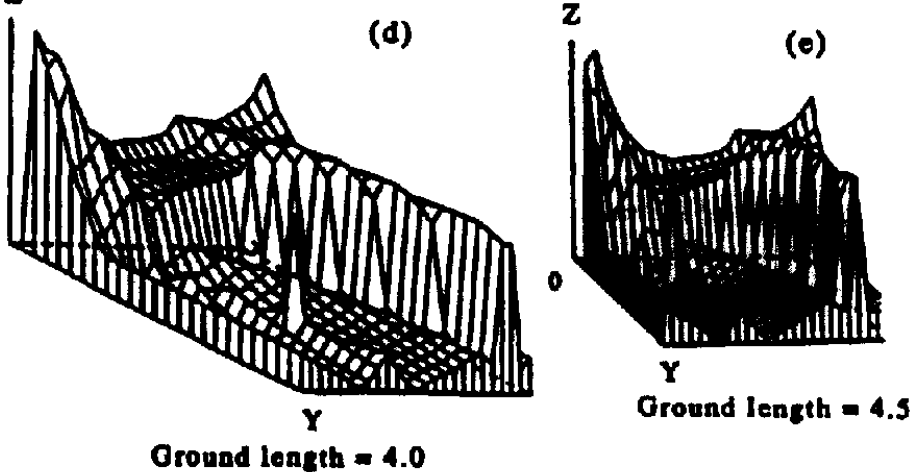

Fig. 6. (Caption below.)

(a)

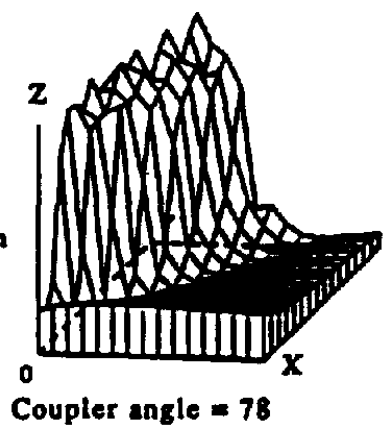

(d)

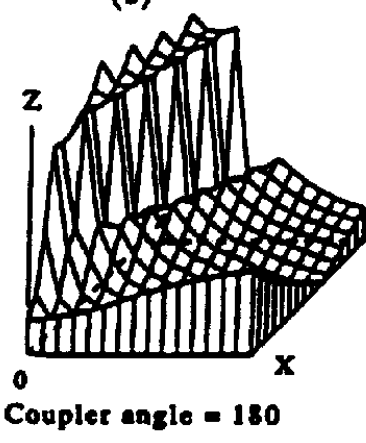

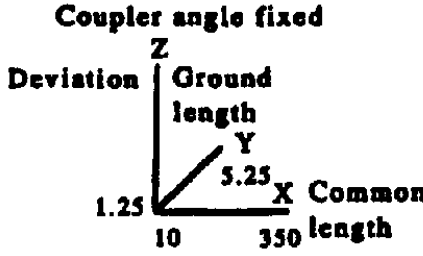

Coupler angle $=78$

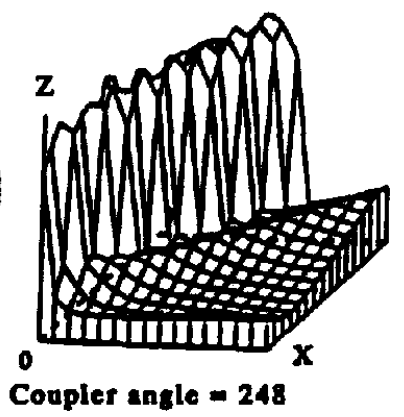

(b)

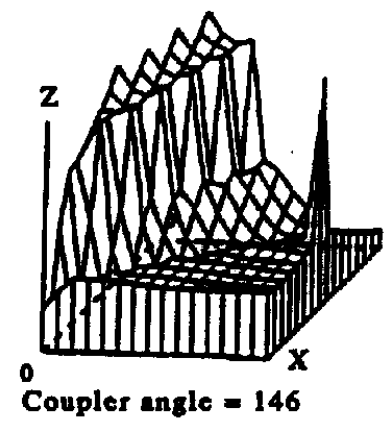

(e)

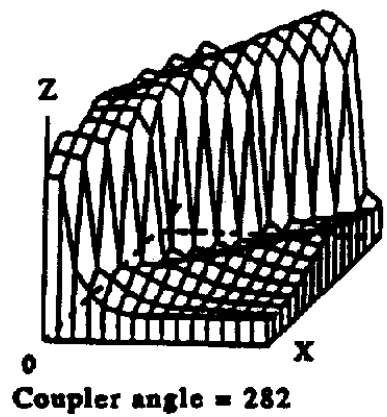

Fig. 7

Figs 5-7. Typical contour plots of the objective function for mechanism path generation. These figures indicate the behavior of the objective function as two of the three design parameters are varied keeping the third fixed at difierent values. In Fig. 5, common length is held fixed, in Fig. 6 ground length is held fixed and in Fig. 7 coupler angle is held fixed. 
and 4.5 units. Figure 7 corresponds to cases where the coupler angle is fixed at $78,146,180,248$, and $282^{\circ}$.

Figures 5-7 clearly indicate the existence of multiple local minima and the nonmonotonic nature of the objective function. Our investigations have also revealed that the coupler angle has the most significant influence on the shape of the coupler curve and hence on the value of the objective function. The abrupt peaks in the objective function values in the middle of the design planes correspond to coupler curves with double points. A visual display of the objective function behavior as a function of design variables provides the necessary insight into the design problem to identify appropriate search techniques. Certain points in the design space yield link dimensions for which a linkage cannot be assembled. The objective function is not evaluated at these points. This is another source of non-smoothness in the objective function.

Figures 5-7 illustrate the difficulty one would face in identifying a single optimization technique that is suitable for a wide range of path generation problems. Generally, it is difficult to choose a starting point that leads to convergence on global optimum. However, the value of the objective function at some of the local minima is found to be within an acceptable range (less than 0.075 ). A careful examination of the contour plots in Figs 5-7 reveal some acceptable local minima. It can also be seen that the "acceptable" local minima occur at widely different points in the design space. This is encouraging since a starting point near any of the "acceptable" minima could lead to a satisfactory solution. Although, convergence on the global minimum is not necessary. convergence on at least one of the "acceptable" local minima still poses enough challenge in this highly nonlinear domain. In the next section, we will investigate the use of statistically designed experiments as an alternative to traditional optimization methods for mechanism design.

\section{EXPERIMENTAL DESIGN}

Experimental design is a well-known technique used in off-line quality control. The use of statistically designed experiments enables one to achieve product or process development objectives with far fewer resources. In the case of product development, typically, a set of design variables $x_{1}, x_{2}, \ldots, x_{n}$ that influence the performance of the product are identified. These are controllable factors. They are controllable in the sense that the designer through engineering judgement, would assign specific values to these variables so that the resulting product would have optimum performance. However, there are usually other factors over which the designer has no control but which, nonetheless, affect the product's performance. These are uncontrollable factors or noise factors. In order to optimize the performance of a product under given constraints, it is important to understand the effect of each design variable on the performance of the product. It is also important to design a product that is insensitive to variations in the noise factors, whenever

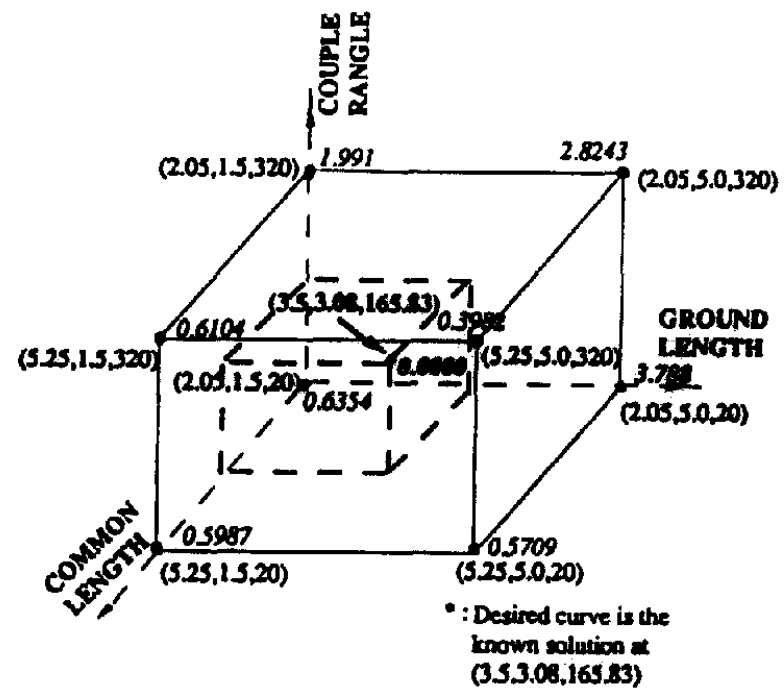

Fig. 8. Three dimensional solution space. Coordinates (a. b. c) correspond to: (common length, ground length. coupler angle) and deviation values are shown in italic. 
possible. The designer's task is to identify optimum values for each controllable factor (design variable) so that the performance of the product remains optimum under different operating conditions or insensitive to noise factors. The traditional approach to experimental design is to vary one factor at a time, holding all others fixed. Statistically designed experiments, on the other hand, allow one to simultaneously vary several factors at a time thus reducing the number of experiments that are required to optimize the product's performance. One such technique is the use of orthogonal arrays and signal-to-noise ratios. Numerous text books [Phadke 1989; Ross 1988] and research articles are available on this subject. In this section, we will introduce the basic terminology for readers who are unfamiliar with this subject, and in the next section, we will demonstrate the use of experimental design techniques in mechanism design problems.

Conducting a designed experiment using special matrices called orthogonal arrays allows us to determine the effects of several design variables efficiently. Table 1 shows a hypothetical orthogonal array. The rows in Table 1 indicate individual experiment runs and the columns represent factors or design variables to be studied. Each design variable can take several values or levels. Thus, experiment number 3 is conducted with the design variable $a$ at level $1, b$ at level $3, c$ at level 3 and so on. The output is either measured experimentally or computed if an analytical formulation exists. The output corresponds to the value of the objective function. Interactions between different factors can be studied using linear graphs [Taguchi 1989] and are not discussed here. Depending on the number of factors, number of levels for each factor, and so on, an appropriate orthogonal matrix can be selected from a library of statistically designed matrices. These matrices are called orthogonal because the columns are pair-wise orthogonal; that is, for every pair of columns, all combinations of factor levels of the respective variables occur exactly once.

The region formed by the factors being studied and their alternate levels is called the experimental region. The values used for factors before conducting a matrix experiment are the starting values. The main effects of the factors are their separate effects. In addition to the array shown in Table I for studying the effects of controllable factors, an additional array, called an outer array, is typically used to study the effect of noise factors. A designer would arbitrarily select typical values (levels) for noise factors and include them in the outer array.

\section{PATH GENERATION USING EXPERIMENTAL DESIGN TECHNIQUES}

In our design example, we have used three design variables (common length, ground length, and coupler angle) as controllable factors. In the example given below, noise factors are not taken into account. A standard orthogonal array that accommodates up to four factors with three levels for each factor is used. This is called the $L_{\text {g }}$ array (Table 2 ) since it requires only nine experiments.

Table I. An orthogonal array (L9)

\begin{tabular}{|c|c|c|c|c|c|}
\hline $\begin{array}{c}\text { Exph } \\
\text { No. }\end{array}$ & $\begin{array}{c}\text { Coupled } \\
\text { Angle } \\
1\end{array}$ & $\begin{array}{c}\text { Ground } \\
\text { Length } \\
2\end{array}$ & $\begin{array}{c}\text { Common } \\
\text { Length }\end{array}$ & 4 & Devinioa \\
\hline 1 & 1 & 1 & 1 & & D1 \\
\hline 2 & 1 & 2 & 2 & & D2 \\
\hline 3 & 1 & 3 & 3 & & D3 \\
\hline 4 & 2 & 1 & 2 & & D4 \\
\hline 5 & 2 & 2 & 3 & & D5 \\
\hline 6 & 2 & 3 & 1 & & D6 \\
\hline 7 & 3 & 1 & 3 & & D \\
\hline 8 & 3 & 2 & 1 & & D \\
\hline 9 & 3 & 3 & 2 & & D9 \\
\hline
\end{tabular}


Table 2. L9 orthogonal array applied to mechanism design problem

\begin{tabular}{|r|r|c|c|c|c|}
\hline $\begin{array}{r}\text { Expl. } \\
\text { No. }\end{array}$ & $\begin{array}{c}\text { Compler } \\
\text { Alezhe } \\
1\end{array}$ & $\begin{array}{c}\text { Growad } \\
\text { Length }\end{array}$ & $\begin{array}{c}\text { Commeo } \\
\text { Longth } \\
3\end{array}$ & 4 & $\begin{array}{c}\text { Deviation } \\
\mathrm{Di}\end{array}$ \\
\hline 1 & 20 & 1.5 & 2.05 & & $\mathrm{D} 1=0.6354$ \\
\hline 2 & 20 & 3.25 & 3.65 & & $\mathrm{D} 2=0.5791$ \\
\hline 3 & 20 & 5.0 & 5.25 & & $\mathrm{D} 3=0.5709$ \\
\hline 4 & 170 & 1.5 & 3.65 & & $\mathrm{D} 4=0.7073$ \\
\hline 5 & 170 & 3.25 & 5.25 & & $\mathrm{D} 5=0.2839$ \\
\hline 6 & 170 & 5.0 & 2.05 & & $\mathrm{D} 6=2.1178$ \\
\hline 7 & 320 & 1.5 & 5.25 & & $\mathrm{D} 7=0.3815$ \\
\hline 8 & 320 & 3.25 & 2.05 & & $\mathrm{D} 8=1.705$ \\
\hline 9 & 320 & 5.0 & 3.65 & & $\mathrm{D} 9=0.4127$ \\
\hline
\end{tabular}

The experimental region is defined by the following bounds: coupler angle (lower bound $20^{\circ}$ and upper bound $320 \%$, ground length (lower bound 1.5 units and upper bound 5.0 units), common length, (lower bound at 2.05 units and upper bound at 5.25 units). The starting values are shown in Table 3.

In experiment I (Table 2), all the three factors are held at level I; that is, coupler angle at $20^{\circ}$, ground length at 1.5 units, and common length at 2.05 units. The performance measure in our path generation task is the value of the objective function; the deviation of the generated curve from the desired curve. The goal is to determine the factor levels that yield minimum deviation. The value of the objective function (deviation) is denoted by $D$, for experiment $i$ and is shown in the last column. Data analysis involves determination of effects of factor levels on the deviation value. The effect of a factor level is determined by summing up the values of the objective function for those experiments in which the factor was set to that level. For instance, consider the control factor "common length." This factor was set at level 1 (i.e. common length $=2.05$ units) in experiments 1,6 , and 8 . The objective function values are $D_{1}, D_{6}$, and $D_{8}$. The effect of level 1 is therefore $D I+D 6+D 8$. Similarly, the effect of level 3 of ground length is equal to $D_{2}+D_{3}+D_{y}$, since the ground length was set to level 3 in experiments 2,5 , and 8 . The level that yields the smallest summations are retained, and new values for the other two levels are defined as follows:

If level 1 yields the smallest deviation (summation of three deviation values), then

(level 1$)_{\text {new }}=(\text { level } 1)_{\text {otd }}$

(level 2) new $=0.5^{*}\left((\text { level } 1)_{\text {new }}+(\right.$ level 3) new $)$

(level 3$)_{\text {new }}=($ level 2) old

Table 3. Starting values (intervals) of control factors define the experimental region

\begin{tabular}{|l|c|c|c|}
\hline Larameter & 1 & 2 & 3 \\
\hline $\begin{array}{l}\text { Coupler } \\
\text { Angle }\end{array}$ & 20 & 170 & 320 \\
\hline $\begin{array}{l}\text { Ground } \\
\text { Length }\end{array}$ & 1.5 & 3.25 & 5.0 \\
\hline $\begin{array}{l}\text { Common } \\
\text { Length }\end{array}$ & 2.05 & 3.65 & 5.25 \\
\hline
\end{tabular}


That is, two new kevels are defined in the neighborhood of the level that yields minimum deviation.

If kevel 2 yields the smallest deviation, then

$(\text { level } 1)_{\text {wew }}=(\text { level } 2)_{\text {odd }}-\left(\right.$ level 2) odd $\left.-(\text { level 1 })_{\text {otd }}\right)$

(level 2) $=($ level 2) odd

(level 3) $)_{\text {ove }}=(\text { level } 2)_{\text {odd }}+\left((\text { level } 3)_{\text {odd }}-(\text { level 1 })_{\text {odd }}\right)$

If level 3 yields the smallest deviation, then

(level 1)

(level 2) $)_{\text {new }}=0.5^{*}\left((\text { level } 1)_{\text {new }}+(\text { level 3) })_{\text {new }}\right)$

(level 3) $)_{\mathrm{osw}}=($ level 3) odd

Another set of nine experiments are conducted with these new levels. The process is repeated until

(a) the deviation value is less than or equal to 0.01 , or

(b) the maximum difference in the effects of levels is less than 0.005 for each control factor. This implies that the objective function reached a valley.

After nine iterations, the deviation value was found to be 0.0093 with corresponding factor levels of:

common length $=4.25$ units,

ground length $=3.25$ units,

coupler angle $=100.8^{\circ}$.

Note that the desired solution (deviation value $=0.000$ ) is at common length $=3.5$ units, ground length $=3.08$ units and the coupler angle $=165.83^{\circ}$. The experimental design procedure did not give us the global minimum but converged on an acceptable local minimum (deviation less than 0.01 ) in only nine iterations. This translates to 81 function evaluations since there are nine experiments per iteration. It will be shown later that the solution obtained here, when used as an initial guess in traditional optimization method leads to a better solution, in this case, the correct solution.

\section{Noise factors}

The goal is to minimize the value of the objective function by controlling the values of the design variables. However, uncontrollable factors (noise) such as manufacturing errors cause the value of the output variable to deviate from its nominal value. This nominal value or the mean value of the output variable, is achieved by setting the design variables at their "optimum" values. The goal then would be to identify the values of design variables such that the average value of the output is closer to the target value and the variation in the target value is minimized; that is, the variation in the output is less sensitive to noise. Let $\mathrm{X}=\left(x_{1}, x_{2}, x_{3} \ldots, x_{n}\right)^{T}$ denote the noise factors and $Z=\left(z_{1}, z_{2}, z_{3}, \ldots, z_{4}\right)^{T}$ denote the design variable or control factors whose values are set by the designer. The product's quality characteristic $y$ may be a complex nonlinear function of $X$ and $Z$, i.e. $y=f(X, Z)$. The principal goal of robust design is to find combinations of $Z$ values that give the desired target value of the product characteristic under nominal noise conditions (when the noise factors are exactly their nominal values). The deviation $D_{y}$ of the quality characteristic from the target value caused by the deviation $D_{x t}$ of the noise factors from their respective nominal values can be approximated as

$\Delta Y=\left[\delta f / \delta_{x 1}\right] \Delta_{x 1}+\cdots+\left[\delta f / \delta_{x+n}\right] \Delta_{x m}$ noise factors are uncorrelated, the variance, $\sigma_{y}^{2}$, of $y$ can be expressed in terms of variances, $\sigma_{x}^{2}$ of the individual noise factors as $\sigma_{y 2}=\left[\delta f / \delta_{x 1}\right]^{2} \sigma_{x 1}^{2}+\cdots+\left[\delta f / \delta_{x n}\right]^{2} \sigma_{x n}^{2}$.

The sensitivity coefficients, $\left[\delta f / \delta_{x 1}\right]^{2}$, are themselves functions of the control factor values. A robust product is one for which the sensitivity coefficients are the smallest.

The quality characteristic in our case is the deviation of the generated coupler curve from the desired curve. Here, the quality characteristic is continuous and nonnegative. Its most desired value, however, is zero. Such problems are called "smaller-the-better" type problems. Literature in robust design and Taguchi methods discusses other types such as "nominal-the-best" and 
"larger-the-better" type problems as well. Loss in quality oocurs whenever the output value does not coincide with the desired target value. Taguchi developed the "loss function" to quantify losses due to variations in the product performance caused by noise factors. Quality loss for the smaller-the-better type problems is given by

$$
Q=k\left[(1 / n) \Sigma y_{i}^{2}\right]
$$

where the term in the brackets is the mean square quality characteristic, $k$ is a constant, $n$ is the number of experiments, and $y_{i}$ is the measured value of the quality characteristic.

Minimizing the quality loss, $Q$, is equivalent to maximizing $S / N$, the signal-to-noise ratio, which is defined by the following equation

$$
\mathrm{S} / \mathrm{N}=-10 \log _{10}\left[(1 / n) \Sigma y_{i}\right]
$$

$\mathrm{S} / \mathrm{N}$ is called the signal-to-noise ratio. In the above expression for $\mathrm{S} / \mathrm{N}$, note that we have ignored the constant $k$ and expressed the quality loss in decibels. Using the $S / N$ ratio rather than the mean square deviation from target as an objective function has several advantages. The interested reader is encouraged to refer to literature in robust design [Phadke 1989] for further details on $\mathrm{S} / \mathrm{N}$ ratios.

\section{Design example using solerances}

In this design example, variations in link lengths due to tolerances, joint clearances, and link deflections are represented as noise factors. The noise factors are included in an outer array. An $L_{4}$ orthogonal array has been selected to represent noise factors. Three noise factors (coupler angle variation, common length variation, and ground length variation) with two levels per factor are represented in the $L 4$ array. The nominal values of the coupler angle, common length, and ground length are represented as levels in the main array (as in previous example). In order to set the levels in the outer array, we have arbitrarily assumed $\pm 5 \%$ variation in coupler angle, and $\pm 1 \%$ variation in the other two factors. That is, the design experiment is carried out with an assumption that theoretical link length could vary as much as $\pm 1 \%$ due to manufacturing tolerances, joint clearances, and link deflections. This being a preliminary study, the effects of tolerances (which are random), joints clearances and link deflections (which are load dependent) are lumped together even though identical treatment of them is not very realistic. The experimentil region in this example is the same as used in the previous case.

The matrix experiment is shown in Table 4. Since variations in link lengths can occur randomly in practice, it is important that our designed experiment is carried out for different combinations of such variations. In experiment 1, for instance, all the design variables are set at their first levels (coupler angle $20^{\circ}$, ground length 1.5 units, and common length 2.05 units), and the deviation values are computed for different combinations of noise factors. The third measurement in experiment $I$ has the coupler angle variation set at level $2\left(-5 \%\right.$ of $20^{\circ}$ or $-1^{\circ}$ variation), ground length variation at level $1(+1 \%$ of 1.5 units or 0.015 units), and common length variation at level 2 ( $-1 \%$ of 2.05 units or -0.0205 units). Thus, four measurements per experiment are noted, and the $S / N$ ratio is computed using equation (1), where $n$ is the number of measurements per experiment ( $n=4$ in our case), $y_{i}$ are the measured values and these correspond to the measured deviation values. Table 5 shows the inner and outer arrays and signal-to-noise ratios of the first iteration for this example.

The procedure to identify new levels for control factors that are needed for successive experiments is given below.

Step 1. Determine the influence of each level for every control factor.

Control factor 1: Common length

Effect of level $1=$ SN1 + SN6 + SN8

Effect of level $2=$ SN2 + SN4 + SN9

Effect of level $3=\mathrm{SN} 3+\mathrm{SN} 5+\mathrm{SN} 7$

Control factor 2: Ground length

Effect of level $1=\mathrm{SN} 1+\mathrm{SN} 4+\mathrm{SN} 7$

Effect of level $2=\mathrm{SN} 2+\mathrm{SN} 5+\mathrm{SN} 8$

Effect of level $3=$ SN3 + SN6 + SN9 
Table 4. Inner and outer arrays. Controllable factors and their levels occupy the inner array. Noise factors, which are deliberately introduced, and theit kevels are shown in the outer array

\begin{tabular}{|c|c|c|c|c|c|c|c|c|}
\hline \multicolumn{3}{|c|}{ Outer Array - LA $\rightarrow$} & Coupler & 1 & 1 & 2 & 2 & \\
\hline Inner Ar & $\mathrm{ray}-\mathrm{L}$ & & Ground & 1 & 2 & 1 & 2 & \\
\hline & & & Length & 1 & 2 & 2 & 1 & \\
\hline $\begin{array}{l}\text { Expt. } \\
\text { No. }\end{array}$ & $\begin{array}{l}\text { Couple } \\
\text { Angle }\end{array}$ & $\begin{array}{l}\text { Ground } \\
\text { Length }\end{array}$ & $\begin{array}{l}\text { Common } \\
\text { Length }\end{array}$ & & & & & S/N \\
\hline 1 & 1 & 1 & 1 & DI & 02 & 03 & D4 & SN 1 \\
\hline 2 & $\mathfrak{l}$ & 2 & 2 & DS & D6. & D7 & DB & SN 2 \\
\hline 3 & $\mathbf{1}$ & 3 & 3 & Q & D10 & 011 & 012 & SN 3 \\
\hline 4 & 2 & 1 & 2 & 013 & 014 & 015 & 016 & SN 4 \\
\hline$s$ & 2 & 2 & 3 & 017 & 018 & 019 & $\mathbf{0 2 0}$ & SN 5 \\
\hline 6 & 2 & 3 & 1 & 021 & 022 & $\mathrm{D} 23$ & 024 & $5 N 6$ \\
\hline 7 & 3 & 1 & 3 & 025 & 026 & D27 & D28 & SN 7 \\
\hline 8 & 3 & 2 & 1 & 029 & 030 & 031 & 032 & SN 8 \\
\hline 9 & 3 & 3 & 2 & 033 & 034 & DJS & 036 & SN 9 \\
\hline
\end{tabular}

Tuble S. Mechanism design using inner and outer arrays and signal-to-

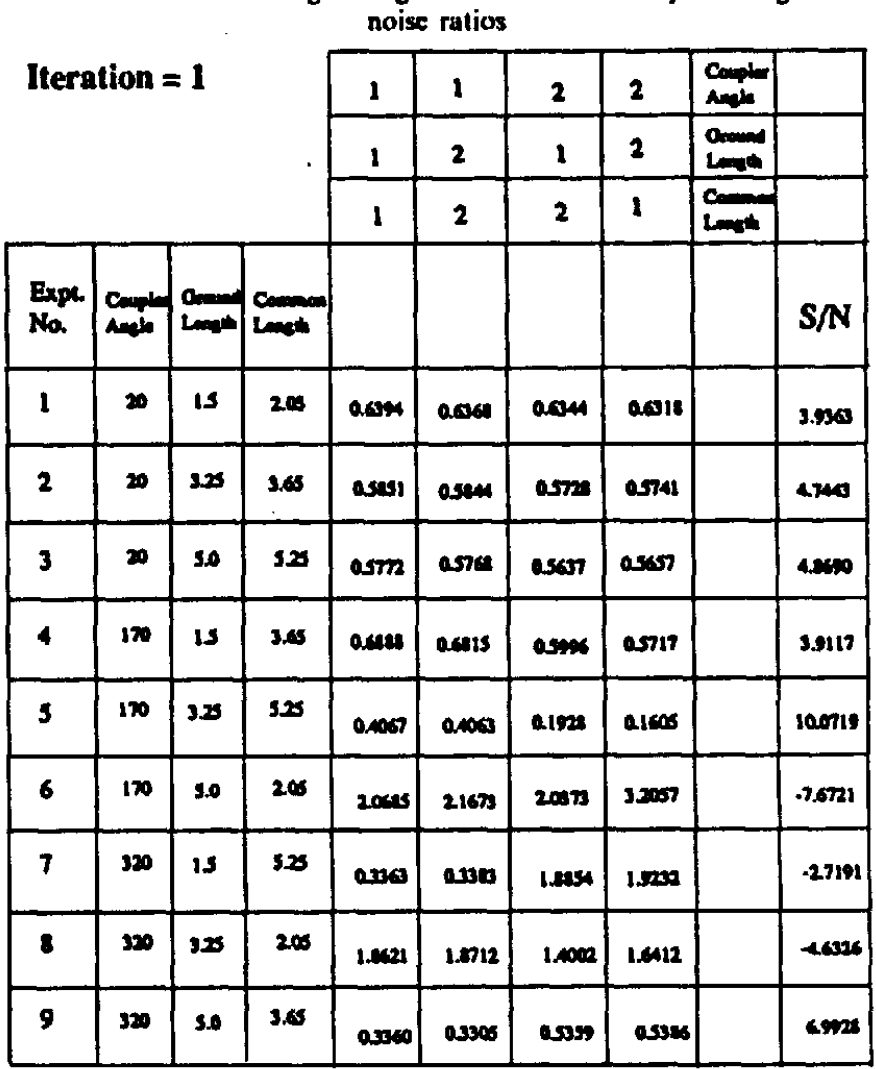


Control factor 3: Coupler angle

Effect of level $1=\mathrm{SN} 1+\mathrm{SN} 2+\mathrm{SN} 3$

Effect of level $2=$ SN4 + SN5 + SN6

Effect of level $3=$ SN7 + SN8 + SN9

Step 2. Identify the level for each control factor that had the most influence on SN values. That is, choose the level that produced the largest sum since we are interested in maximizing the $\mathrm{S} / \mathrm{N}$ ratio.

Step 3. Define the values for new levels. This is done by picking values in the neighborhood of the level identified in step 2. The value of the level identified in step 2 does not change.

If level 1 had the largest sum, then

(level 1$)_{\text {new }}=(\text { level } 1)_{\text {okd }}$

$(\text { level } 2)_{\text {new }}=\left((\text { level } 1)_{\text {new }}+(\text { level } 3)_{\text {new }}\right) / 2$

(level 3) $)_{\text {new }}=(\text { level } 2)_{\text {old }}$

If level 2 had the largest sum, then

(level 2) $)_{\text {new }}=($ level 2) old

$(\text { level } 1)_{\text {new }}=(\text { level } 2)_{\text {ovd }}-\left((\text { level } 2)_{\text {odd }}-(\text { level } 2)_{\text {odd }}-(\text { level } 1)_{\text {old }}\right)$

$(\text { level } 3)_{\text {new }}=(\text { level } 2)_{\text {old }}+\left(\right.$ old level $\left.2-(\text { level } 1)_{\text {old }}\right)$

If level 3 had the largest sum, then

(level 3) $)_{\text {new }}=($ level 3) oud

(level 1) $)_{\text {new }}=($ level 2) old

$(\text { level } 2)_{\text {new }}=\left((\text { level 1 })_{\text {new }}+(\text { level } 3)_{\text {new }}\right) / 2$

Step 4. Repeat the matrix experiment until

(a) the deviation value $(D j)$ is less than 0.075 , or

(b) the effect of a control factor (max. difference in effect of its levels) is less than 0.005 . This implies that the search has reached a valley.

After 10 iterations (see Table 6), or 360 function evaluations (36 evaluations per iteration) the deviation value was found to be $\mathbf{0 . 0 6 2}$. The link dimensions are:

common length $=4.412( \pm 1 \%)=4.412 \pm 0.44$

ground length $=3.687( \pm 1 \%)=3.687 \pm 0.036$

coupler angle $=96.162^{\circ}( \pm 5 \%)=(96.16 \pm 4.81)^{\circ}$.

Once again, the solution procedure has converged on a local minimum. It should be noted that the experimental region covered the entire design space. Most importantly, all the link variations due to tolerances, clearances, and so forth are taken into account in the design procedure.

In the next design example, we have selected a smaller experimental region in the neighborhood of the global solution. The new experimental region is shown in Table 7. As expected, the solution procedure converged very close to the global solution with 0.0009 deviation in only six iterations. The link dimensions are:

Common length $=\mathbf{3 . 4 8}$ units,

Ground length $=3.015$ units,

Coupler angle $=167.18^{\circ}$.

\section{INITIAL GUESS IN A TRADITIONAL OPTIMIZATION METHOD}

In order to compare and improve upon the results obtained by using the experimental design method with the results obtained using a conventional optimization method, the same planar path generation problem is considered. The optimization algorithm used is the Generalized Reduced Gradient (GRG) method [Gabriele and Ragsdell, 1977]. The GRG method is an efficient and robust algorithm that can handle complex nonlinear optimization problems. The objective function is 
Table 6. Results obtained on the tenth iteration

\begin{tabular}{|c|c|c|c|c|c|c|c|c|c|}
\hline & & & & 1 & 1 & 2 & 2 & 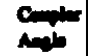 & \\
\hline & & & & 1 & 2 & 1 & 2 & $\stackrel{0}{1}$ & \\
\hline & & & & 1 & 2 & 2 & 1 & (1) & \\
\hline $\begin{array}{l}\text { Expl. } \\
\mathrm{Na} .\end{array}$ & مانس & | & canted & & & & & & $\mathbf{S} / \mathbf{N}$ \\
\hline 1 & ssons & 3.020 & AN128 & 0.1000 & $\operatorname{acose}$ & a.me & armin & & 21.103 \\
\hline 2 & 95870 & $3 \cos 1$ & astse & 0.1000 & 0.0531 & a.0.41 & a.on! & & $21.1 \operatorname{sen}$ \\
\hline 3 & ss.mp, & $20 \mathrm{nts}$ & Aa187 & $a \mathrm{arn}$ & aosese & o.pat & 2.07n1 & & 21.171 \\
\hline 4 & x.17ns & 3.60 & AA1S6 & acmon & $0.0 \mathrm{~m} 1$ & o.om & $0.00 \mathrm{n}$ & & 21.1522 \\
\hline 5 & $x<17 n$ & $3 . \mathrm{sen} 1$ & ansen & $0.0 \mathrm{~m}$ & acose & 0070 & 20012 & & 21.1603 \\
\hline 6 & «1ng & $3 . \mathrm{cm}$ & AA125 & a.om & 0.007 & 0.006 & a.coned & & $21.1 \mathrm{~mm}$ \\
\hline 7 & $x \rightarrow 40$ & 3.600 & 4A18 & $\cos 8$ & Dosos & 0007 & 0.000 & & 21.1059 \\
\hline 8 & races & 3.001 & AAIZS & acoss & 0.050 & 0.078 & 0.0048 & & 21.1000 \\
\hline 9 & rancen & sens & 4.136 & a.cosen & 0.009 & o.me & and & & 21.175 \\
\hline
\end{tabular}

formulated as the sum of squares of structural errors (SSSE), as is usually done in the optimal synthesis of mechanisms. Thus the objective function here is different from the metric used in the experimental method. The term structural error is interpreted as the straight line distance between the desired and the obtained points. Mathematically the objective function is represented as below.

$$
f(X)=\sum_{j=1}^{n}\left\{\left(\boldsymbol{P}_{j x}-\bar{R}_{j x}\right)^{2}+\left(\boldsymbol{F}_{j y}-\bar{R}_{j y}\right)^{2}\right\}
$$

where $\boldsymbol{X}$ is the array of unknown variables and $\bar{P}$ and $R$ are the obtained and the desired position vectors of the tracer points.

A four-bar mechanism that is required to pass through 20 data points, as closely as possible, is synthesized. Another difference between this and the problem treated in the experimental design method is that, here, each point is associated with a particular rotation of the input crank from its initial position, i.e. with prescribed input timing. The procedure used to compute the deviation in the experimental design method does not account for input timing.

For comparison purposes, the 20 data points are generated (as against specifying on the basis of an arbitrary path to be traced in an application) with the same mechanism parameters as

Table 7. Starting values (intervals) of control factors

\begin{tabular}{|c|c|c|c|}
\hline Level & 1 & 2 & 3 \\
\hline Conplex Angle & 150 & 175 & 200 \\
\hline Ground Length & 2.5 & 3.25 & 4.0 \\
\hline Common Length & 2.5 & 3.25 & 4.0 \\
\hline
\end{tabular}


were used in illustrating the behavior of the objective function and later in the experimental method. A uniform spacing with an increment of $18^{\circ}$ has been used to generate the data. By specifying the upper and lower limits on all the variables, the objective function is minimized using an optimization routine that uses the GRG algorithm. The results of two cases with different numbers of variables are provided in Table 8 . In the first case, only three variables (ground link length, common link length, and coupler angle) are considered. In the second case, seven linkage parameters are varied. The four additional variables are the coordinates of the input and output crank pivots. As these four determine the ground link length, it is no more an additional parameter. Instead, the input crank length is treated as a variable. Common link length and coupler angle are the two other variables. The optimum values obtained for six arbitrary initial design points in each case are given in Table 8.

It can be seen, in Table 8, that the minimum objective function value for the three-variable case, at the end of convergence of the algorithm is very close to zero in four cases, but in cases 2 and 5 , the optimum value obtained is 103.4287 , which is a local minimum (see Figs 5-7). On the other hand, in the seven-variable case, an acceptable local minimum can be obtained only if the design point is in the vicinity of the solution. Thus, the chances of converging upon an acceptable local minimum are heavily dependent upon the initial guess. By comparing the two test cases, it can be concluded that the greater the number of variables, the more complex the design space and consequently, the difficulties with unacceptable local minima and sensitivity to initial guess are

Table 8. Results from GRG method

\begin{tabular}{|c|c|c|}
\hline \multicolumn{3}{|c|}{$X=$ (fround link length $(x 1)$, common link length $(x 2)$ and coupler angle $(x 3))$} \\
\hline \multirow[b]{2}{*}{ No. } & $\bar{x}$ & SSSE* \\
\hline & $\begin{array}{l}\text { Iritial Davien Poins } \\
\text { Final Desion Point }\end{array}$ & $\begin{array}{l}\text { Initial } \\
\text { Optimum }\end{array}$ \\
\hline 1 & $\left\{\begin{array}{l}2.0,8.0,150.0\} \\
3.06,3.490,166.089\}\end{array}\right.$ & 0.0021 \\
\hline 2 & 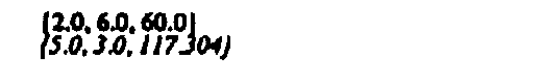 & $\begin{array}{l}1107.4389 \\
103.4287\end{array}$ \\
\hline 3 & $\left\{\begin{array}{l}4.0,8.0,135.0\} \\
3.096,3,501,165916\}\end{array}\right.$ & $\begin{array}{l}1690.8452 \\
0.0020\end{array}$ \\
\hline 4 & $\{3.0 .3 .0,150.0\}$ & 30.8266 \\
\hline $\mathbf{s}$ & $\left\{\begin{array}{l}4.0,8.0,45.0 \\
5.0 .3 .0 .117,301\}\end{array}\right.$ & $\begin{array}{l}1191.5689 \\
103.4287\end{array}$ \\
\hline 6 & $\{3.05 i .3 .0,2709.0\}$ & $\begin{array}{l}\$ 38.9881 \\
0.0054\end{array}$ \\
\hline 7 & 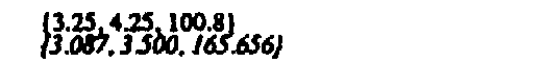 & 0.0005 \\
\hline 8 & $\{3.697,4.412,96.16)$ & $\begin{array}{l}358.3926 \\
0.0010\end{array}$ \\
\hline \multicolumn{3}{|c|}{ 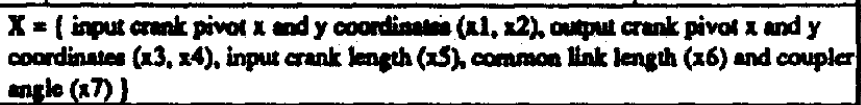 } \\
\hline 1 & 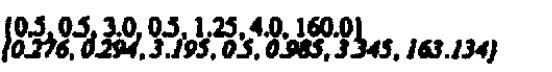 & $\begin{array}{l}81.2900 \\
0.0457\end{array}$ \\
\hline 2 & $\left(\begin{array}{l}-2.0,3.0,5.0,2.0,2.0,6.0,225,0) \\
-2.617,2.678,0562,3,419,1 . J 31,3,280,219.641)\end{array}\right.$ & $\begin{array}{l}670.0914 \\
18.8063\end{array}$ \\
\hline 3 & 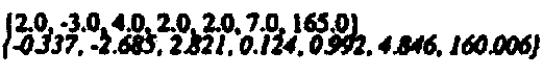 & 552.3045 \\
\hline 4 & $\left\{\begin{array}{l}2.0,5.0,3.0,2.0,20,400,8.0 \\
2,024,5968,3.181 .1 .057,0.5,2.73,8.858\}\end{array}\right.$ & 156.6641 \\
\hline 5 & $\left\{\begin{array}{l}20,3.0,-3.0,0.0,3.25,65,100.0 \\
3.036, i 388,-3560,-0324,1039,4.415,61276)\end{array}\right.$ & 943.6134 \\
\hline 6 & 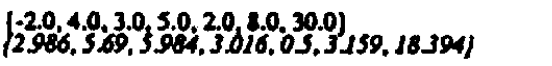 & $\frac{1229.5117}{77.7135^{17}}$ \\
\hline
\end{tabular}


increased if we use a traditional optimization method. Cases 7 and 8 in the three variables case use the results of experimental method as initial guesses. It can be seen that the solution obtained is almost zero and the design point obtained is very close to the known solution for the path generation problem considered in this work.

\section{CONCLUSIONS AND FUTURE WORK}

This paper is based on our preliminary investigations into the applicability of experimental design techniques to mechanism design tasks. The design examples given in this paper clearly indicate the potential applications of orthogonal arrays in solving path generation problems. It is also important to note that the notion of noise factor captures the link-length variations due to tolerances, clearances, and link deformations. Kinematic solutions that are robust against such noise factors are identified by using both inner and outer arrays. Experimental design techniques are easy to use and are computationally inexpensive since they are based on simple linear search and evaluation of the objective function. The design examples indicate that neither the experimental design procedure nor the GRG optimization scheme converged on the global minimum. However, as depicted in Figs 5-7, there are usually several acceptable local minima that both solution procedures are capable of converging upon. It should further be noted that the solution of experimental method, when used as an initial guess for the optimization method led to the globally optimal solution very closely.

This paper is only a first word in the use of experimental design procedures to search for mechanism solutions. It is not the intent of this paper to prove the superiority of one technique over another. Experimental design procedures only consume less resources. As in anything else, there is always a tradeoff between complexity and efficiency. Since the efficiency of most mathematical optimization techniques depend upon the starting point, it might be worthwhile to use orthogonal arrays to identify at least a good starting point. Given a good starting point, one of many optimization schemes that are capable of converging upon an acceptable local minima could then be used. We plan to investigate, in future, some of the well-established "optimal design of experiments" procedures [Ferorov 1972] to solve mechanism design tasks.

Acknowledgements - Authors wish to thank Dr Selden Crary. Electrical Engineering and Computer Science Department. University of Michigan, for his input on advanced visualization techniques.

\section{REFERENCES}

Erdman A. G. (Ed.), The First Forty Years of Modern Kinematics, Proceedings of the "Modern Kinematics Conference-A Tribuse to Ferdinand Freudenstein" (1991). Minneapolis, July (Book, 13 Chaps). In press.

Faik S. and Erdman A. G., Sensitivity distribution in the synthesis solution space of four-bar linkages. Truns. ASME J. Mech. Design 113 (No. 1), 309 (March 1991).

Ferorov V. V., Theory of Optimal Experiments. Academic Press, New York (1972).

Gabriele G. A. and Ragedell K. M., The generalized reduced gradient method: A reliable tool for optimal design. ASME J. Engng Industry 99, 394-400 (1977).

Garrett R. E. and Hall A. S., Effect of tolerance and clearance in linkage design. Trans. ASME J. Engng Industry (February), 198-202 (1969).

Jensen P., Synthesis of four-bar linkages with a coupler curve passing through 12 points. Mech. Mach. Theory 19 (No. I). $149-165$ (1984).

Jensen P., The inverted coupler curve method for path generation. IOh Applied Mechanisms Conference (1987).

Kinzel G. L. and A. S. Hall, Tolerances and clearances in instrumented spatial chains. Proc. Inst. Mech. Engrs $185-191$ (1975).

Knappe L. F., A technique for analyzing mechanism tolerances. Mach. Design 25, 155-157 (April 1963).

Kota S., Automatic selection of mechanism designs from a three-dimensional design map. J. Mech. Design. In press.

Kramer S. and Sandor G. N., Selective precision synthesis-A general method of optimization for planar mechanisms. ASME Trans. J. Engng Industry Ser. B 97 (No. 2), 689-701 (May 1975).

Phadke M. S., Qwality Engineering Using Robust Design. Prentice-Hall, NJ (1989).

Ros P. J., Taguchi Techniques for Quality Engineering, Loss Function, Orthogonal Experiments, Parameter and Tolerance Design. McGraw-Hill, New York (1988).

Roth, A generalization of Burmester theory: Nine-point path generation of geared five-bar mechanisms with gear ratio plus and minus one. Dissertation, Columbia University (1962).

Sharfi $O$. M. and Smith M. R., A simple method for the allocation of appropriate tolerances and clearances in linkage mechanisms. Mech. Mach. Theory 18 (No. 2). 123-129 (1983).

Taguchi G., Introduction to Quality Engineering. Asian Productivity Center (1989). 
Resul-[l n'existe pas une seul méthode d'optimisation mieux adaptè à resoudre éffectivement tous les problemes de la ginération des trajets (Erdman 1991). Des graphiques qui montrent l'espace du dessin du mécanisme figurent dans cet exposé: ils font preuve de la difficulté que l'on rencontre ì optimiser les tiches de generation des trajets. Un exemple a été également inclus afin d'illustrer l'importance d'une bonne conjecture de base pour la réalisation d'une conversion correcte. Cet exposé propose l'utilisation des techniques de dessin expérimentales que sont fondées sur une base des rangs orthogonaux statistiquement dessinés, puisque ces techniques de dessin sont vus comme une méthode alternative pour la résolution des problèm de dessin des mécanismes. Lutilisation de cette méthode serait également bénéfique quand on cherche a obtenir un bon point de départ pour les meilleures procédures possibles de synthèse traditionnelle. Au moment de la traduction d'une solution théorique en réalité physique. la performance d'un mécanisme peut varier à cause des tolérances de fabrication, des dégagements de jointure, et de l'élasticité des membres chainons. Les rangs orthogonaux. et les procédures robustes de dessin qui y sont alliées, proposés ici. peuvent aussi expliquer ces variations. Des exemples de dessins sont préséntés pour illustrer l'utilisation des rangs orthogonaux. 